\title{
A Novel Information Fusion Method for Redundant Rotational Inertial Navigation Systems Based on Reduced-Order Kalman Filter
}

\author{
Lin Wang ${ }^{1}$, Wenqi $\mathrm{Wu}^{1}$, Guo Wei ${ }^{1}$, Jinlong $\mathrm{Li}^{2}$ and Ruihang $\mathrm{Yu}^{1}$ \\ ${ }^{1}$ National University of Defense Technology, 410073 Changsha, China \\ ${ }^{2} 92330$ Troops of People's Liberation Army, Production Department, 266102 Qingdao, China
}

\begin{abstract}
The redundant rotational inertial navigation systems can satisfy not only the high-accuracy but also the high-reliability demands of underwater vehicle on navigation system. However, different systems are usually independent, and lack of information fusion. A reduced-order Kalman filter is designed to fuse the navigation information output of redundant rotational navigation systems which usually include a dual-axis rotational inertial navigation system being master system and a single-axis rotational inertial navigation system being hot-backup system. The azimuth gyro drift of single-axis rotational inertial navigation system can be estimated by the designed filter, whereby the position error caused by that can be compensated with the aid of designed position error prediction model. As a result, the improved performance of single-axis rotational inertial navigation system can guarantee the position accuracy in the case of dual-axis system failure. Semi-physical simulation and experiment verify the effectiveness of the proposed method.
\end{abstract}

\section{Introduction}

The modern inertial navigation system (INS) should satisfy not only the high-accuracy but also the highreliability demands of underwater vehicle during longendurance sailing trial [1]. Rotational inertial navigation system (RINS) based on optical gyro has been proven to meet the high-accuracy demand of underwater vehicle [2]. In order to satisfy the high-reliability demand, redundant RINS configuration is usually adopted by underwater vehicle [3].

RINS can average out the influence of gyro drifts and accelerometer biases with the aid of gimbals [4-6]. The redundant RINS configuration including a dual-axis RINS and a single-axis RINS is a good scheme which makes trade-off among the accuracy, the reliability and the cost. The dual-axis RINS can average out all the gyro drifts and accelerometer biases, and the performance of it mainly depends on gyro angular random walk $[4,5]$. Hence, the dual-axis RINS is with the higher accuracy, and usually as master INS in spite of its lower reliability and higher cost in comparison to single-axis RINS. On the other hand, the single-axis RINS has the advantages of the higher reliability and the lower cost regardless of its poorer performance in comparison to dual-axis RINS, which makes it an excellent backup system for dual-axis RINS [6]. However, information fusion between the two systems is usually ignored, and the systems generally run independently from one another.

Aimed at solving the above problem, an augmented error states Kalman filter is designed to estimate the inertial sensor deterministic errors of the redundant RINSs, whereby a prediction model is established to estimate the position error of single-axis RINS caused by its gyro drifts and accelerometer biases [7]. As a result, the position output from single-axis RINS after position error compensation is still at high level even if the dualaxis RINS fails. However, since all the inertial sensor deterministic errors except for the single-axis RINS azimuth gyro drift can be averaged out, it is not necessary to take them as error states, which will relax computation burden.

In this paper, without using external information, a reduced-order Kalman filter is designed for the redundant RINS configuration to estimate the azimuth gyro drift of single-axis RINS. And on this basis, a prediction model is established to predict the position error of single-axis RINS caused by azimuth gyro drift. After compensation for the position error, the single-axis RINS is comparable in position accuracy with the dual-axis RINS. Finally, semi-physical simulation and experiment are conducted to verify the effectiveness of the proposed method.

\section{Reduced-order Kalman filter design}

\subsection{Error states dynamic model}

An augmented error states Kalman filter is designed for redundant RINS configuration in [7], where the attitude error's difference, the velocity error's difference and the position error's difference between the single-axis RINS 
1 and the dual-axis RINS 2 as well as the gyro drifts and accelerometer biases are taken as error states. The augmented error state vector is given by

$$
\mathbf{x}(t)=\left[\begin{array}{lllllll}
\phi_{12}^{n} & \delta \mathbf{v}_{12}^{n} & \delta \mathbf{p}_{12}^{n} & \boldsymbol{\varepsilon}_{1}^{b_{1}} & \boldsymbol{\varepsilon}_{2}^{b_{2}} & \nabla_{1}^{b_{1}} & \nabla_{2}^{b_{2}}
\end{array}\right]^{T}
$$
with

$$
\begin{aligned}
& \phi_{12}^{n}=\phi_{1}^{n}-\phi_{2}^{n}, \delta \mathbf{v}_{12}^{n}=\delta \mathbf{v}_{1}^{n}-\delta \mathbf{v}_{2}^{n}, \delta \mathbf{p}_{12}^{n}=\delta \mathbf{p}_{1}^{n}-\delta \mathbf{p}_{2}^{n} \\
& \boldsymbol{\varepsilon}_{1}^{b_{1}}=\left[\begin{array}{llll}
\varepsilon_{x}^{b_{1}} & \varepsilon_{y}^{b_{1}} & \varepsilon_{z}^{b_{1}}
\end{array}\right]^{T}, \mathbf{\varepsilon}_{2}^{b_{2}}=\left[\begin{array}{lll}
\varepsilon_{x}^{b_{2}} & \varepsilon_{y}^{b_{2}} & \varepsilon_{z}^{b_{2}}
\end{array}\right]^{T} \\
& \nabla_{1}^{b_{1}}=\left[\begin{array}{lll}
\nabla_{x}^{b_{1}} & \nabla_{y}^{b_{1}}
\end{array}\right]^{T}, \nabla_{2}^{b_{2}}=\left[\begin{array}{lll}
\nabla_{x}^{b_{2}} & \nabla_{y}^{b_{2}}
\end{array}\right]^{T}
\end{aligned}
$$

where $\phi_{12}^{n}$ denotes the difference between the attitude error of single-axis RINS 1, i.e., $\phi_{1}^{n}$, and that of dual-axis RINS 2, i.e., $\phi_{2}^{n} ; \delta \mathbf{v}_{12}^{n}$ denotes the difference between the velocity error of single-axis RINS 1, i.e., $\delta \mathbf{v}_{1}^{n}$, and that of dual-axis RINS 2, i.e., $\delta \mathbf{v}_{2}^{n} ; \delta \mathbf{p}_{12}^{n}$ denotes the difference between the position error of single-axis RINS 1, i.e., $\delta \mathbf{p}_{1}^{n}$, and that of dual-axis RINS 2, i.e., $\delta \mathbf{p}_{2}^{n} ; \boldsymbol{\varepsilon}_{1}^{b_{1}}$ and $\boldsymbol{\varepsilon}_{2}^{b_{2}}$ respectively denote the gyro triad drift of singleaxis RINS 1 and dual-axis RINS $2 ; \nabla_{1}^{b_{1}}$ and $\nabla_{2}^{b_{2}}$ denote the accelerometer biases of the two systems. Note that the error states related with vertical channel are ignored, e.g., the vertical velocity error's difference, the altitude error's difference, and the vertical accelerometer biases. The position error are in the form of latitude error and longitude error. Corresponding error state vector dynamic model can be expressed by

$$
\dot{\mathbf{x}}(t)=\mathbf{F}(t) \mathbf{x}(t)+\mathbf{G}(t) \mathbf{w}(t)
$$

with

$$
\mathbf{w}(t)=\left[w_{\varepsilon_{x}}^{b_{1}}, w_{\varepsilon_{y}}^{b_{1}}, w_{\varepsilon_{z}}^{b_{1}}, w_{\varepsilon_{x}}^{b_{2}}, w_{\varepsilon_{y}}^{b_{2}}, w_{\varepsilon_{z}}^{b_{2}}, w_{\nabla_{x}}^{b_{1}}, w_{\nabla_{y}}^{b_{1}}, w_{\nabla_{x}}^{b_{2}}, w_{\nabla_{y}}^{b_{2}}\right]^{T}
$$

where $\mathbf{w}(t)$ is the process nosie, and the system matrix $\mathbf{F}(t)$ and process noise matrix $\mathbf{G}(t)$ are given in [1].

In fact, all the inertial sensor deterministic errors except for the azimuth gyro drift of single-axis RINS 1, i.e., $\varepsilon_{z}^{b_{1}}$, can be averaged out by rotating the inertial mearuement unit (IMU) of RINS periodically. It is not necessary to take them as error states, so the error state vector in Eq. (1) can be reduced to a reduced-order error state vector as

with

$$
\mathbf{x}_{r}(t)=\left[\begin{array}{llll}
\phi_{12}^{n} & \delta \mathbf{v}_{12}^{n} & \delta \mathbf{p}_{12}^{n} & \varepsilon_{z}^{b_{1}}
\end{array}\right]^{T}=\mathbf{T} \mathbf{x}(t)
$$

$$
\mathbf{T}=\left[\begin{array}{c:c}
\mathbf{I}_{7} & \mathbf{0}_{7 \times 10} \\
\hdashline \mathbf{0}_{1 \times 7} & \mathbf{T}_{22}
\end{array}\right], \mathbf{T}_{22}=\left[\begin{array}{lll:l}
0 & 0 & 1 & \mathbf{0}_{1 \times 7}
\end{array}\right]
$$

Corresponding dynamic model of reduced-order error state vector is given by

$$
\dot{\mathbf{x}}_{r}(t)=\mathbf{F}_{r}(t) \mathbf{x}_{r}(t)+\mathbf{G}_{r}(t) \mathbf{w}(t)
$$

with

$$
\mathbf{F}_{r}(t)=\mathbf{T F}(t) \mathbf{T}^{T}, \mathbf{G}_{r}(t)=\mathbf{T G}(t)
$$

where the components of $\mathbf{F}_{r}(t)$ and $\mathbf{G}_{r}(t)$ are given by

$$
\mathbf{F}_{r}(t)=\mathbf{T F}(t) \mathbf{T}^{T}=\left[\begin{array}{ccc:c}
\mathbf{F}_{1} & \mathbf{F}_{2} & \mathbf{F}_{3} & \mathbf{C}_{b_{1}}^{n} \mathbf{N} \\
\mathbf{F}_{4} & \mathbf{F}_{5} & \mathbf{F}_{6} & \mathbf{0}_{2 \times 1} \\
\mathbf{0}_{2 \times 3} & \mathbf{F}_{7} & \mathbf{F}_{8} & \mathbf{0}_{2 \times 1} \\
\hdashline & \mathbf{0}_{1 \times 7} & & 0
\end{array}\right], \mathbf{N}=\left[\begin{array}{c}
0 \\
0 \\
-1
\end{array}\right]
$$

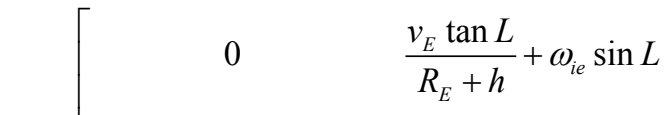

$$
\begin{aligned}
& \mathbf{F}_{1}=\left[\begin{array}{cc}
-\frac{v_{E} \tan L}{R_{E}+h}-\omega_{i e} \sin L & 0 \\
\omega_{i e} \cos L+\frac{v_{E}}{R_{E}+h} & \frac{v_{N}}{R_{N}+h}
\end{array}\right. \\
& \left.\begin{array}{c}
-\omega_{i e} \cos L-\frac{v_{E}}{R_{E}+h} \\
-\frac{v_{N}}{R_{N}+h} \\
0
\end{array}\right], \mathbf{F}_{2}=\left[\begin{array}{cc}
0 & -\frac{1}{R_{N}+h} \\
\frac{1}{R_{E}+h} & 0 \\
\frac{\tan L}{R_{E}+h} & 0
\end{array}\right] \\
& \mathbf{F}_{3}=\left[\begin{array}{cc}
0 & 0 \\
-\omega_{i e} \sin L & 0 \\
\omega_{i e} \cos L+\frac{v_{E}}{\left(R_{E}+h\right) \cos ^{2} L} & 0
\end{array}\right] \\
& \mathbf{F}_{4}=\left[\begin{array}{ccc}
0 & -f_{U} & f_{N} \\
f_{U} & 0 & -f_{E}
\end{array}\right] \\
& \mathbf{F}_{5}=\left[\begin{array}{cc}
\frac{v_{N} \tan L}{R_{E}+h} & \frac{v_{E} \tan L}{R_{E}+h}+2 \omega_{i e} \sin L \\
-2 \omega_{i e} \sin L-2 \frac{v_{E} \tan L}{R_{E}+h} & 0
\end{array}\right] \\
& \mathbf{F}_{6}=\left[\begin{array}{cc}
2 \omega_{i e} v_{N} \cos L+\frac{v_{N} v_{E}}{\left(R_{E}+h\right) \cos ^{2} L} & 0 \\
-2 \omega_{i e} v_{E} \cos L-\frac{v_{E}^{2}}{\left(R_{E}+h\right) \cos ^{2} L} & 0
\end{array}\right] \\
& \mathbf{F}_{7}=\left[\begin{array}{cc}
0 & \frac{1}{R_{N}+h} \\
\frac{1}{\left(R_{E}+h\right) \cos L} & 0
\end{array}\right] \\
& \mathbf{F}_{8}=\left[\begin{array}{cc}
0 & 0 \\
\frac{v_{E} \tan L}{\left(R_{E}+h\right) \cos L} & 0
\end{array}\right], \mathbf{M}=\left[\begin{array}{lll}
1 & 0 & 0 \\
0 & 1 & 0
\end{array}\right] \\
& \mathbf{G}_{r}(t)=\left[\begin{array}{cc:c}
-\mathbf{C}_{b_{1}}^{n} & \mathbf{C}_{b_{2}}^{n} & \mathbf{0}_{3 \times 4} \\
\hdashline \mathbf{0}_{2 \times 6} & \mathbf{M} \mathbf{C}_{b_{1}}^{n} \mathbf{M}^{T}-\mathbf{C}_{b_{2}}^{n} \mathbf{M}^{T} \\
\hdashline & \mathbf{0}_{3 \times 10}
\end{array}\right]
\end{aligned}
$$

The navigation frame is local level geographic frame (East-North-Up). The body frames of single-axis RINS 1 and dual-axis RINS 2 are $b_{1}$ and $b_{2}$, respectively. $v_{E}$ and $v_{N}$ are true east velocity and north velocity of underwater vehicle, respectively. $\omega_{i e}$ is the earth rotation rate. $L$ and $h$ are true latitude and height. $R_{E}$ and $R_{N}$ are transverse radius of curvature and meridian radius curvature, respectively. $f_{E}, f_{N}$ and $f_{U}$ are respectively the east, the north and the up component of specific force. 


\subsection{Observation model}

As shown in Figure 1, the velocity outputs and position outputs from single-axis RINS 1 and dual-axis RINS 2 meet the following relationships

$$
\begin{aligned}
\tilde{\mathbf{p}}_{1}^{n}=\mathbf{p}^{n}+\delta \mathbf{p}_{1}^{n}, & \tilde{\mathbf{p}}_{2}^{n}=\mathbf{p}^{n}+\mathbf{p}_{12}^{n}+\delta \mathbf{p}_{2}^{n} \\
\tilde{\mathbf{v}}_{1}^{n}=\mathbf{v}^{n}+\delta \mathbf{v}_{1}^{n}, & \tilde{\mathbf{v}}_{2}^{n}=\mathbf{v}^{n}+\boldsymbol{\omega}_{b_{1} b_{2}}^{n} \times \mathbf{p}_{12}^{n}+\delta \mathbf{v}_{2}^{n}
\end{aligned}
$$

where $\tilde{\mathbf{p}}_{1}^{n}$ and $\tilde{\mathbf{p}}_{2}^{n}$ respectively denote the position output of single-axis RINS 1 and dual-axis RINS 2, $\tilde{\mathbf{v}}_{1}^{n}$ and $\tilde{\mathbf{v}}_{2}^{n}$ respectively denote the velocity output of them; $\mathbf{p}^{n}$ and $\mathbf{v}^{n}$ respectively denote the true position and true velocity of underwater vehicle; $\mathbf{p}_{12}^{n}$ denotes the lever-arm between the single-axis RINS 1 and dual-axis RINS 2, which can be accurately calibrated in advance. The operator ' $\delta$ ' denotes the error perturbation of corresponding variables.

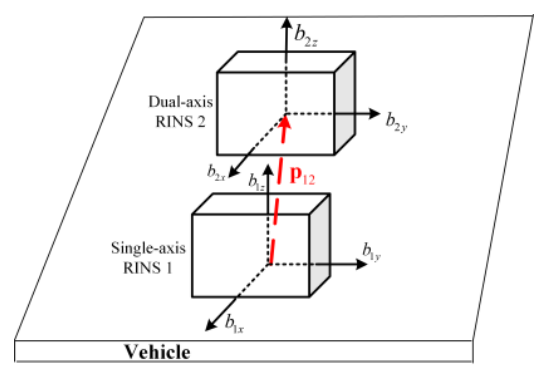

Figure 1. Redundant RINS configuration.

Subtracting the second equation of Eq. (19) and (20) from the first one yields

$$
\begin{aligned}
& \delta \mathbf{p}_{12}^{n}=\delta \mathbf{p}_{1}^{n}-\delta \mathbf{p}_{2}^{n}=\tilde{\mathbf{p}}_{1}^{n}-\tilde{\mathbf{p}}_{2}^{n} \\
& \delta \mathbf{v}_{12}^{n}=\delta \mathbf{v}_{1}^{n}-\delta \mathbf{v}_{2}^{n}=\tilde{\mathbf{v}}_{1}^{n}-\tilde{\mathbf{v}}_{2}^{n}
\end{aligned}
$$

where the influence of lever-arm is ignored. Hence, the observation model of reduced-order error state vector is given by

$$
\mathbf{z}(t)=\mathbf{H}_{r}(t) \mathbf{x}_{r}(t)+\mathbf{v}(t)
$$

with

$$
\begin{aligned}
& \mathbf{z}(t)=\left[\begin{array}{llll}
\delta v_{E 12} & \delta v_{N 12} & \delta L_{12} & \delta \lambda_{12}
\end{array}\right]^{T} \\
& \mathbf{H}_{r}(t)=\left[\begin{array}{ccc}
\mathbf{I}_{2} & 0_{2 \times 2} & 0_{2 \times 3} \\
0_{2 \times 2} & \mathbf{I}_{2} & 0_{2 \times 3}
\end{array}\right]
\end{aligned}
$$

where $\delta v_{E 12}$ and $\delta v_{N 12}$ are the east component and north component of $\delta \mathbf{v}_{12}^{n} ; \delta L_{12}$ and $\delta \lambda_{12}$ are the latitude error's difference and longitude error's difference between single-axis RINS 1 and dual-axis RINS 2; v(t) is observation noise.

\subsection{Observability analysis}

Since the system $\left(\mathbf{F}_{r}(t), \mathbf{H}_{r}(t)\right)$ satisfies the definition of piece-wise constant system, the stripped observability matrix can be used to analyse its observability [8]. The stripped observability matrix is given by

$$
\mathbf{O}(m)=\left[\begin{array}{l:l:l:l}
\mathbf{O}_{1}^{T} & \mathbf{O}_{2}^{T} & \cdots & \mathbf{O}_{m}^{T}
\end{array}\right]^{T}
$$

$$
\mathbf{O}_{j}=\left[\begin{array}{l:l:l:l}
\mathbf{H}_{r}^{T} & \left(\mathbf{H}_{r} \mathbf{F}_{r}\right)^{T} & \cdots & \left(\mathbf{H}_{r} \mathbf{F}_{r}^{7}\right)^{T}
\end{array}\right]^{T}, j=1,2, \ldots, m
$$

where $m$ is the segment.

The observability analysis is conducted in uniform linear motion scenario for simplicity. Substituting Eqs. (11) and (24) into Eq. (25), and calculating the rank of it yields

$$
\operatorname{rank} \mathbf{O}(1)=8
$$

which means the reduced-order error state vector is always completely observable.

\section{Information fusion method}

As shown in Figure 2, with the aid of reduced-order Kalman filter, the azimuth gyro drift of single-axis RINS 1 can be estimated online, whereby the position error caused by it can be predicted. In normal condition, the dual-axis RINS 2 is usually as master INS and outputs position information. Moreover, once the dual-axis RINS 2 fails, the single-axis RINS 1 will keep on outputting position information after position error compensation. As a result, the performance of single-axis RINS 1 is comparable with that of dual-axis RINS in the case of dual-axis RINS 2 failure.

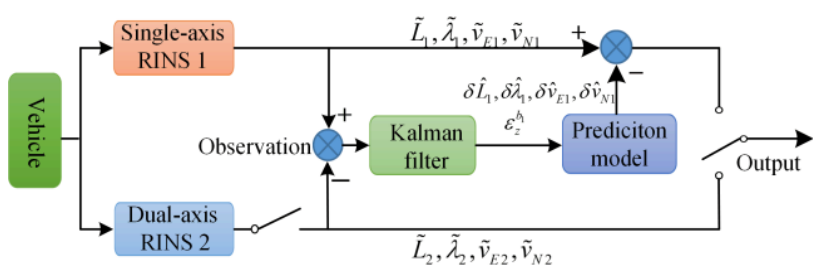

Figure 2. Information fusion method.

The error state vector of single-axis RINS 1 can be expressed as

$$
\mathbf{x}_{1}(t)=\left[\begin{array}{llll}
\phi_{1}^{n} & \delta \mathbf{v}_{1}^{n} & \delta \mathbf{p}_{1}^{n} & \varepsilon_{z}^{b_{1}}
\end{array}\right]^{T}
$$

The dynamic model of above error state vector is given by

$$
\dot{\mathbf{x}}_{1}(t)=\mathbf{A}_{1}(t) \mathbf{x}_{1}(t)+\mathbf{G}_{1}(t) \mathbf{w}_{1}(t)
$$

with

$$
\begin{aligned}
& \mathbf{A}_{1}(t)=\mathbf{F}_{r}(t), \mathbf{G}_{1}(t)=\mathbf{G}_{r}(t) \\
& \mathbf{w}_{1}(t)=\left[\begin{array}{lllll}
w_{\varepsilon_{x}}^{b_{1}} & w_{\varepsilon_{y}}^{b_{1}} & w_{\varepsilon_{z}}^{b_{1}} & w_{\varepsilon_{x}}^{b_{2}} & w_{\varepsilon_{y}}^{b_{2}}
\end{array}\right]^{T}
\end{aligned}
$$

Discretizing the above equation yields the position error prediction model of single-axis RINS 1 as

$$
\hat{\mathbf{x}}_{1}\left(t_{k+1}\right)=\boldsymbol{\Phi}_{1}\left(t_{k+1}, t_{k}\right) \hat{\mathbf{x}}_{1}\left(t_{k}\right)
$$

with

$$
\boldsymbol{\Phi}_{1}\left(t_{k+1}, t_{k}\right)=e^{\mathbf{A}_{1} \Delta t}, \hat{\mathbf{x}}_{1}\left(t_{0}\right)=\mathbf{0}
$$

where $\hat{\mathbf{x}}_{1}\left(t_{k}\right)$ is the error state vector prediction at time $t_{k}$, and its initial value is set as zero vector; $\boldsymbol{\Phi}_{1}\left(t_{k+1}, t_{k}\right)$ is the transition matrix; $\Delta t$ is the discretization step size. Note that only the system error of single-axis RINS 1 caused by azimuth gyro drift can be predicted, and the prediction model does not work on random error caused by white noise.

With the position error prediction value subtracted from the position information output of single-axis RINS

with 
1 as follows, the position accuracy of it can be improved to a certain extent

$$
\begin{aligned}
& \bar{L}_{1}=\tilde{L}_{1}-\delta \hat{L}_{1}, \quad \bar{\lambda}_{1}=\tilde{\lambda}_{1}-\delta \hat{\lambda}_{1} \\
& \bar{v}_{E 1}=\tilde{v}_{E 1}-\delta \hat{v}_{E 1}, \quad \bar{v}_{N 1}=\tilde{v}_{N 1}-\delta \hat{v}_{N 1}
\end{aligned}
$$

where the superscripts ' - ' and ' $\wedge$ ' denote the correction output value and prediction value, respectively.

\section{Experiment and discussion}

\subsection{Semi-physical simulation test}

The single-axis RINS 1 adopts a rotation scheme which includes four dwell positions, and the dual-axis RINS 2 adopts a 16-phase sequence cycle rotation scheme $[5,6]$. The actual inertial sensor noise data reflecting the sensor noise level is added to the simulation data. The gyro angular random walk is less than $0.001 \% \sqrt{h}$, and the power spectral density of accelerometer noise is less than $20 \mu \mathrm{g} / \sqrt{\mathrm{Hz}}$. The constant gyro triad drifts of single-axis RINS 1 are $0.003^{\circ} / \mathrm{h},-0.002^{\circ} / \mathrm{h}$ and $0.0003^{\circ} / \mathrm{h}$, and those of dual-axis RINS 2 are $0.004^{\circ} / \mathrm{h},-0.005^{\circ} / \mathrm{h}$ and $0.003^{\circ} / \mathrm{h}$. The horizontal accelerometer biases of single-axis RINS 1 are $20 \mu \mathrm{g}$ and $-40 \mu \mathrm{g}$, and those of dual-axis RINS 2 are $20 \mu \mathrm{g}$ and $-30 \mu \mathrm{g}$. The simulation time is 120 hours. The assumed dual-axis RINS 2 failure moment is the $16^{\text {th }}$ hour. After that moment, the position output is provided by the single-axis RINS 1 .

As shown in Figure 3, the azimuth gyro estimate error is within its $3 \sigma$ bounds, and the estimate accuracy can achieve $0.00005^{\circ} / \mathrm{h}$. Figure 4 depicts the predictions of latitude error and longitude error. As shown in the figure, the prediction model works well. Figure 5 shows the single-axis RINS' radial position error comparison before and after error prediction compensation. It is found that the position accuracy of single-axis RINS 1 after error compensation is comparable with dual-axis RINS 2.

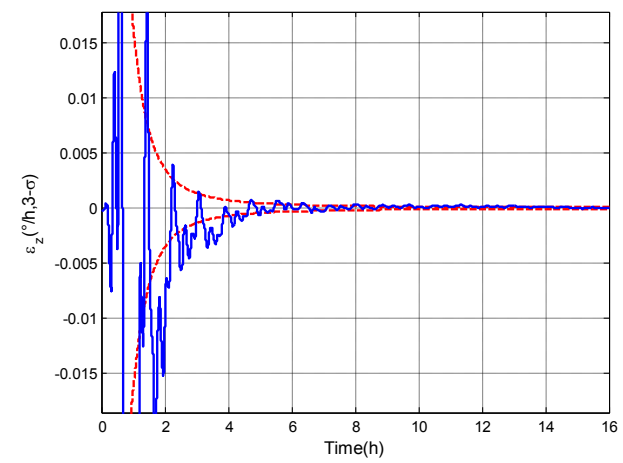

Figure 3. Azimuth gyro drift estimate error and $3 \sigma$ bounds.

Figure 6 shows the radial position error curves of single-axis RINS, which are respectively corresponding to augmented error states Kalman filter and reduced-order error states Kalman filter. As shown in the figure, the information method based on reduced-order Kalman filter is comparable in performance with the information method based on augmented error states Kalman filter.
Hence, even if the dual-axis RINS 2 fails, the position accuracy is still at high level.
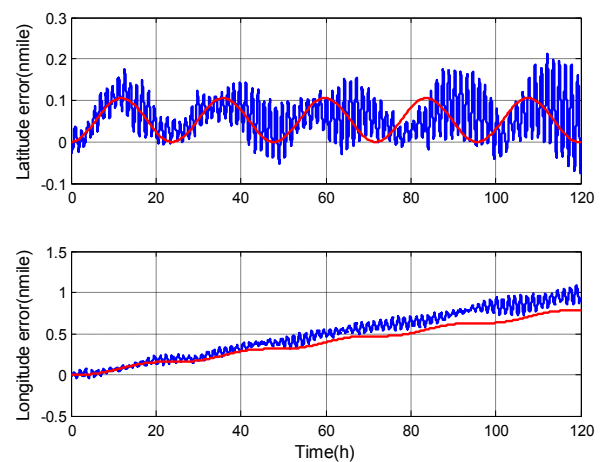

Figure 4. Position error prediction.

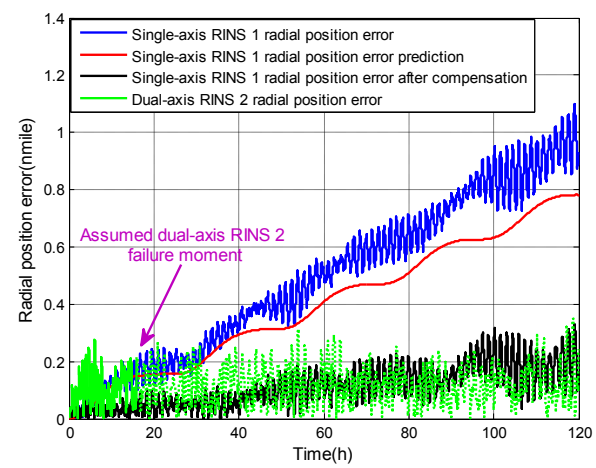

Figure 5. Comparison of position error.

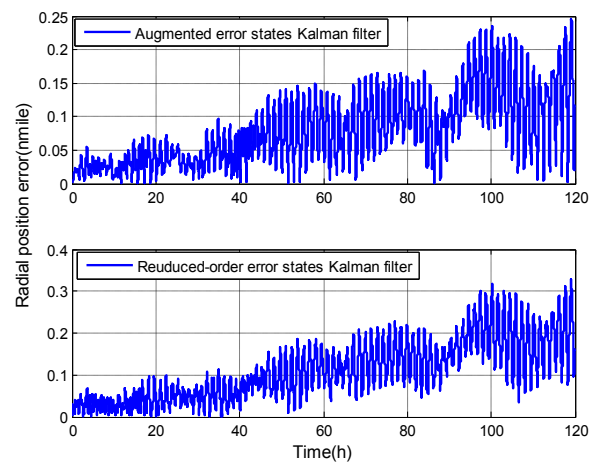

Figure 6. Position error comparison.

\subsection{Lab test}

Lab static test is conducted to verify the effectiveness of proposed method. After self-alignment, both the systems work in pure inertial navigation mode. The assumed dualaxis RINS 2 failure moment is the $24^{\text {th }}$ hour. After that moment, the single-axis RINS 1 outputs position information with error compensation. Figure 7 depicts the predictions of latitude error and longitude error. As expected, the prediction model works well, especially on longitude error. Figure 8 depicts the normalized radial position error comparison of single-axis RINS. After position error compensation, its accuracy is improved to the same level as dual-axis RINS 2. 

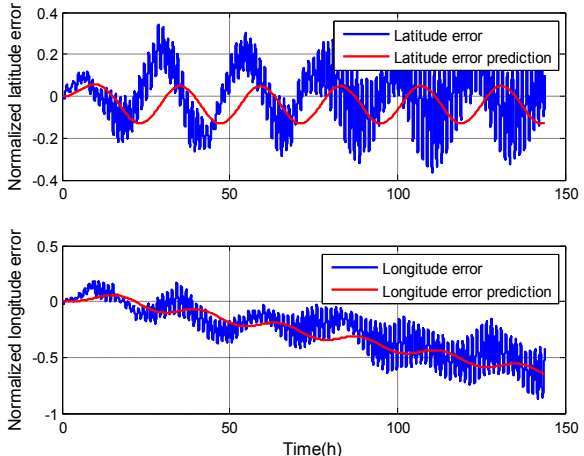

Figure 7. Position error prediction (Lab test).

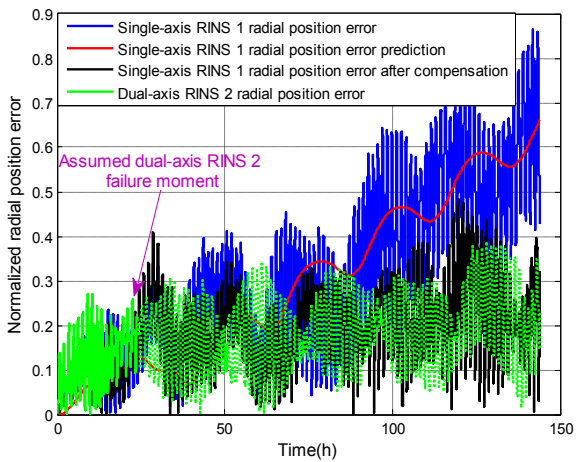

Figure 8. Comparison of position error (Lab test).

\subsection{Sea trial}
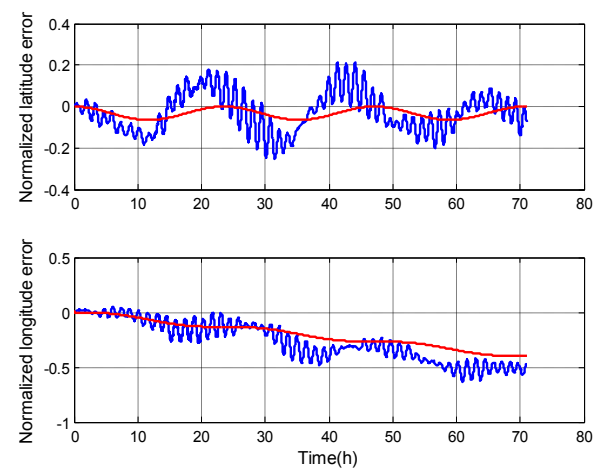

Figure 9. Position error prediction (Sea trial).

A sea trial is conducted to verify the effectiveness of the proposed method. The test time is about 72 hours. Both the systems work in pure inertial navigation mode. GPS position information is used as external reference. The assumed dual-axis RINS 2 failure moment is the $14^{\text {th }}$ hour. Also, after that moment, the single-axis RINS 1 keeps on outputting position information after error compensation. As shown in Figure 9, the position error prediction model works well, especially on longitude error which builds up as time. As depicted in Figure 10, after position error compensation, the position accuracy of single-axis RINS 1 is improved greatly, which is comparable with that of dual-axis RINS 2.

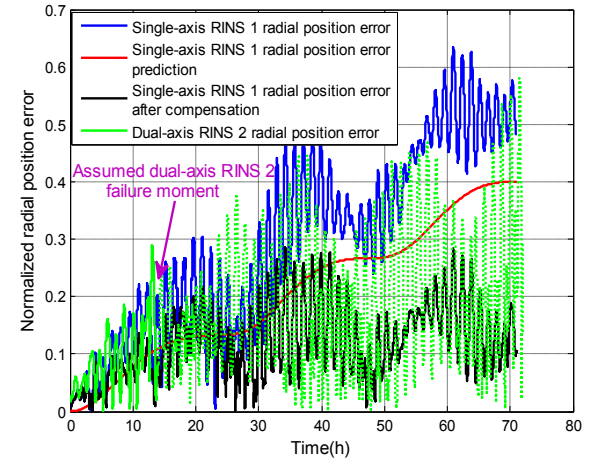

Figure 10. Comparison of position error (Sea trial).

\section{Conclusion}

A novel information fusion method for redundant rotational inertial navigation systems is proposed. A reduced-order Kalman filter is designed to estimate the azimuth gyro drift of single-axis RINS 1. The position error prediction model of single-axis RINS 1 is established to predict its error, and the corresponding position error compensation method is devised. The reduced-order Kalman filter relaxes the computation burden, and is comparable in performance with the augmented Kalman filter. The test results show that the performance of single-axis RINS 1 is improved to the same level as dual-axis RINS 2, which can still guarantee position accuracy in the case of dual-axis RINS 2 failure.

\section{References}

1. D.H. Titterton, J.L. Weston. Strapdown inertial navigation technology (IET, 2004)

2. E. Levinson, C.S. Giovanni. Laser gyro potential for long endurance marine navigation, IEEE Position Location and Navigation Symposium, 115 (1980)

3. E. Levinson, J. Horst, M. Willcocks. The next generation marine inertial navigator is here now, IEEE Position Location and Navigation Symposium (1994)

4. E. Levinson, R. Majure. Accuracy enhancement techniques applied to the marine ring laser inertial navigator (MARLIN), Navigation 34 (1987)

5. B. L. Yuan, D. Liao, S. L. Han. Error compensation of an optical gyro INS by multi-axis rotation. Meas. Sci. Technol. 23 (2012)

6. T. Tucker, E. Levinson. The AN/WSN-7B marine gyroscompass/navigator, Prodeedings of the 2000 National Technical Meeting of the Institute of Navigation (2000)

7. L. Wang, W. Q. Wu, G. Wei, X. F. Pan, C. F. Gao. Dual marine INS joint rotation and modulation for cooperative positioning and error parameter estimation, J. Chin. Inertial Technol. 25 (2017)

8. I. Y. Bar-Itzhack, D. Goshen-Meskin. Observability analysis of piece-wise constant systems-part I: theory. IEEE Trans. on Aerosp. Electron. Syst. 28 (1992) 